\title{
EFECTOS TERRITORIALES DE LAS AUTOPISTAS Y EL TREN DE ALTA VELOCIDAD. EL CASO GALLEGO
}

\author{
Miguel PAZOS OTÓN \\ Departamento de Geografia, Universidad de Santiago de Compostela
}

\section{INTRODUCCIÓN.}

Es indudable que las infraestructuras de transporte constituyen uno de los factores más importantes que debe tener un territorio a priori de cara al desarrollo económico. Numerosos autores (MERLIN, QUINET, PÉREZ TOURIÑO, BIEHL) han estudiado las relaciones que existen entre una buena provisión de infraestructuras de transporte y el crecimiento económico, concluyendo en todos los casos que existe una elevada relación entre ambas, que viene dada por los efectos positivos que supone la mejora de la accesibilidad y la movilidad de personas, mercancías e información dentro del proceso productivo y de las relaciones que éste genera, tanto dentro del espacio de estudio considerado como en las relaciones con el exterior.

Considerando como marco de estudio los países desarrollados, desde un punto de vista estrictamente económico, y en el marco empresarial (QUINET, 1992), la disminución de los tiempos de recorrido entre dos localidades permite el abaratamiento de costes de transporte de mercancías. Los ahorros así conseguidos son susceptibles de ser destinados a labores de investigación, gestión o control de calidad de los productos, lo que asegura una mayor racionalización de las empresas y una mayor aceptación del producto por parte del cliente. Al mismo tiempo, gracias a las mejoras en los tiempos de transporte, es posible aumentar las áreas de mercado y distribución de los productos, lo que genera a su vez beneficiosos efectos económicos que repercuten de nuevo sobre las empresas, aumentando los beneficios.

Desde el punto de vista de la movilidad de personas, las mejoras introducidas en los medios de transporte permiten una más fácil circulación de cuadros directivos, ejecutivos y personal cualificado y técnico, desde los principales centros económicos hacia áreas periféricas de los distintos estados. Esto permite, junto con los grandes avances en la transmisión a distancia y en la gestión de la información, la difusión espacial de las innovaciones a mayor distancia y en mayor grado que antaño.

Así, parece clara la importancia que tienen los sistemas de transporte en la sociedad actual de cara a la movilidad de personas, mercancías e información, lo que ha hecho que algunos autores se refieran a ella como la "sociedad de la movilidad". Aunque las infraestructuras de transporte no son una receta milagrosa que

Polígonos, nº 9, 1999, pp. 227-239. 
asegura sin más el éxito y el desarrollo económico de un territorio, sí son un factor que posibilita y favorece los efectos acumulativos del crecimiento económico. Este debe partir, en todo caso, de un tejido empresarial propio preexistente y desarrollarse posteriormente, mediante la articulación y vertebración interna, y a través de la interacción con los principales centros económicos, de gestión y decisión del exterior, generando la consiguiente movilidad entre distintos territorios. Es a causa de estas necesidades de movilidad donde las infraestructuras de transportes desempeñan un papel más decisivo de cara a las posibilidades de éxito futuras de un territorio determinado.

En este contexto, Galicia, como región periférica dentro de España y Europa (ver FIGURAS 1A y 1B), posee un déficit de infraestructuras de transporte que ha arrastrado a lo largo de su historia y que ha limitado en gran medida sus posibilidades de desarrollo. En efecto, a pesar de la indudable importancia que ha tenido el Camino de Santiago, uno de los más importantes itinerarios terrestres históricos europeos, Galicia ha conocido un secular aislamiento en cuanto a las comunicaciones terrestres con el resto de España y de Europa.

Esta situación comienza a cambiar tímidamente en la década de los 70 , cuando se inicia la construcción de la Autopista del Atlántico, prácticamente finalizada en su totalidad en la actualidad ${ }^{1}$; pero ésta sólo comunica los principales núcleos urbanos del Eje Urbano Atlántico Gallego, principal foco demográfico y verdadero motor económico de Galicia, prolongándose hacia el sur por el dinámico Norte de Portugal, dentro de la Eurorregión Galicia-Norte de Portugal. En cuanto a la comunicación de Galicia con el resto de España y Europa, es en 1984 cuando, dentro del marco del Primer Plan General de Carreteras (1984-1991), se decide y licita la construcción de las dos autovías de enlace con la Meseta (Autovías del Noroeste y de las Rías Baixas), que canalizarán el grueso del tráfico hacia y desde el exterior por carretera una vez finalizadas en su totalidad ${ }^{2}$. Además, desde la descentralización autonómica, y gracias a las competencias adquiridas por el gobierno autonómico de Galicia, se han acometido importantes reformas en la red de carreteras comarcales y locales, lo que ha mejorado mucho la red de carreteras de la Comuni dad en relación con el pasado.

Por lo que se refiere al ferrocarril, la situación actual es muy distinta. El ferrocarril gallego es totalmente decimonónico (NOVOA RODRÍGUEZ, 1991). No existe ningún tramo de vía doble, el porcentaje de vía electrificada es escaso y parámetros como la geometría del trazado en planta (radio de las curvas) o pendientes máximas, son totalmente inadecuados para la moderna explotación ferroviaria y para la adaptación a las demandas de la sociedad actual. Como consecuencia de ello, y a pesar de los tímidos intentos de mejora en la red gallega, Galicia padece un gran déficit de servicio ferroviario, tanto por lo que se refiere a su vertebración interna (con la excepción del saturado eje A Coruña-Santiago-Vigo) como a su comunicación con el exterior, donde se puede afirmar que Galicia está enclavada en términos ferroviarios en relación con el resto de España.

\footnotetext{
${ }^{1}$ A excepción de un pequeño tramo en el acceso a Ferrol.

${ }^{2}$ Resta todavía por finalizar el tramo del Puerto de Pedrafita, en la Autovía del Noroeste.
} 
Figura 1a: Futura Red

\section{Europea de Alta Velocidad Ferroviaria}

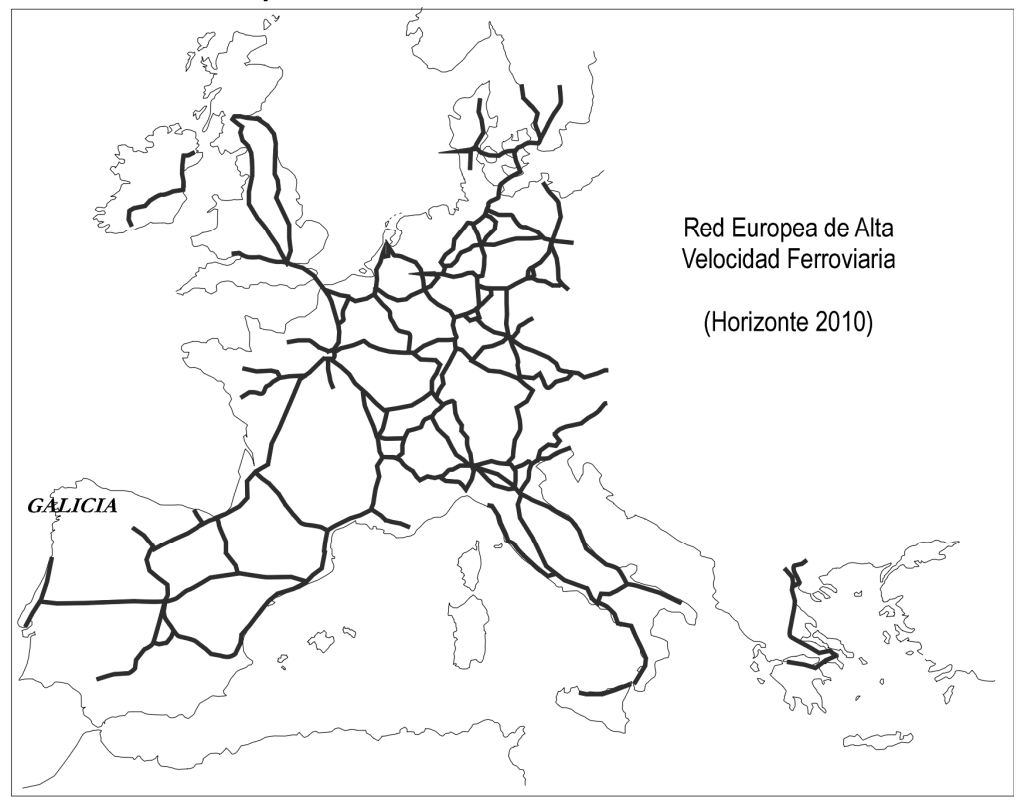

Fuente: Redes Transeuropeas. Comisión Europea (1994)

Figura 1b: Futura Red Europea de Alta Capacidad (Autopistas y Autovías)

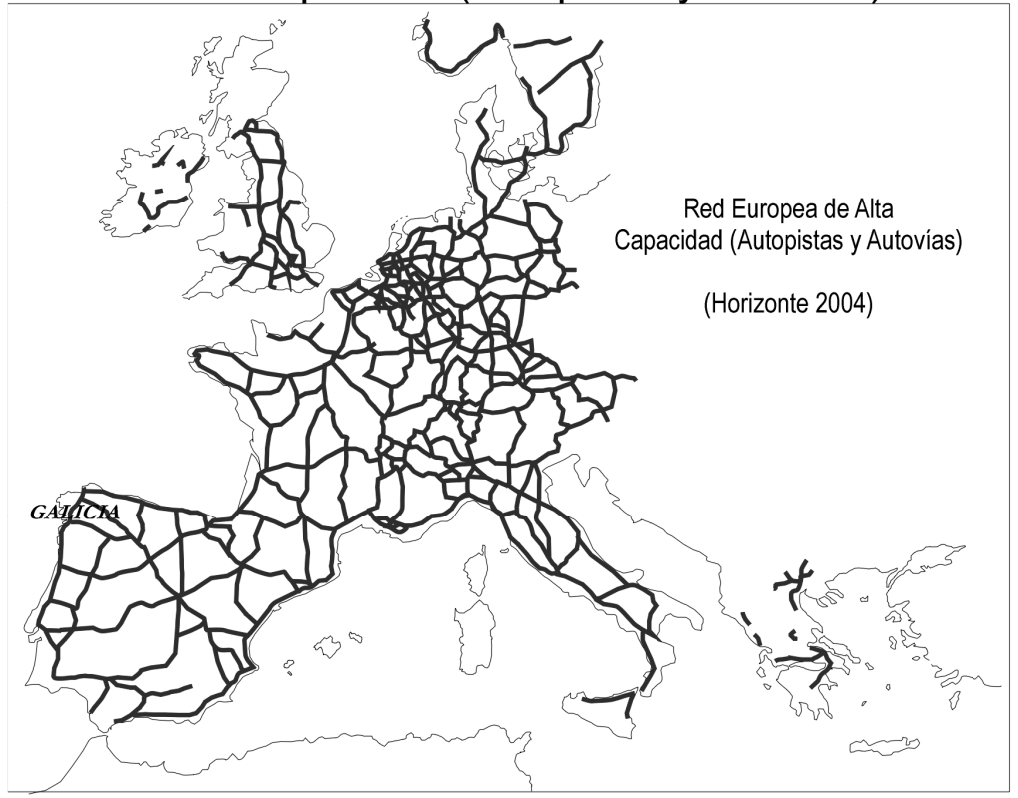

Fuente: Redes Transeuropeas. Comisión Europea (1994) 
En vista de esta situación, nos encontramos, pues, en un momento clave, donde la sociedad en general y los políticos en particular son conscientes de la gran importancia que tienen las infraestructuras en el futuro de Galicia. Existe la sensación generalizada en la sociedad gallega de que el déficit de infraestructuras ha sido uno de los principales factores que han explicado el denominado "atraso gallego" (BEIRAS TORRADO, 1972), y muy poca gente permanece insensible ante la necesidad que tiene Galicia de dotarse unas infraestructuras de transporte modernas y competitivas para poder competir en pie de igualdad con otros territorios.

Estas modernas infraestructuras a las que hacemos alusión son, en la actualidad, las autopistas (autovías), para el caso del transporte por carretera y el tren de alta velocidad (en adelante, TAV), para el transporte ferroviario. A la consecución de las mismas para Galicia se dirigen, por tanto los esfuerzos de políticos y planificadores territoriales. El objetivo prioritario es lograr la conexión con las redes europeas de transporte, tanto en el caso de la carretera como del ferrocarril. Pero debemos prestar atención - y esto no siempre es tenido en cuenta - al papel que deben desempeñar las nuevas infraestructuras de transporte en la vertebración y la cohesión social y territorial interna de Galicia. No es suficiente con crecer y desarrollarse, que vendría materializado por la llegada a Galicia de las modernas infraestructuras de transporte, sino que hay que ordenar racionalmente este crecimiento; la planificación territorial y la ordenación territorial deben proporcionar herramientas para evitar la potenciación de unos preocupantes desequilibrios territoriales que ya existen en Galicia entre el litoral y el interior, que podrían verse agudizados a causa de una incorrecta planificación de las nuevas infraestructuras de transporte, como trataremos de demostrar en el presente texto.

\section{LAS NUEVAS INFRAESTRUCTURAS DE TRANSPORTE. EFEC- TOS SOBRE EL TERRITORIO.}

La sociedad actual, caracterizada por la competición económica, se basa en la necesidad de la rápida movilidad de personas, mercancías e información para conseguir la convergencia espacio-temporal (MASSEY, 1994) y lograr la maximización de beneficios en los intercambios comerciales.

Los medios de transporte, en la actualidad, intentan dar respuesta a estas exigencias y demandas de movilidad, y por ello asistimos hoy en día al auge de dos modos de transporte que reciben toda la atención por parte de políticos y planificadores territoriales: desde el punto de vista de las infraestructuras viarias, las autopistas, y por lo que se refiere a las infraestructuras ferroviarias, el TAV.

Tanto el transporte por autopista como los trenes de alta velocidad son los medios de transporte ya del presente y, sobre todo, del futuro en los países desarrollados, y ofrecen tiempos de viaje realmente competitivos, especialmente en relación con los medios de transporte tradicionales, es decir, la carretera y el ferrocarril convencional. Éstos se encontrarán cada vez más limitados a desplazamientos secundarios de corto alcance o al servicio de áreas periféricas poco dinámicas.

Sin embargo, las características de cada uno de estos dos medios de transporte son muy diferentes, y son asimismo diferentes las consecuencias que tienen sobre el territorio desde el punto de vista del crecimiento económico y el desarrollo te- 
rritorial.

El tren de alta velocidad. Debido a la orientación del TAV casi exclusivamente al transporte de viajeros y su escasa utilización de cara a las mercancías, se configura de este modo como el medio ideal para los flujos de personas, con una clara orientación en el plano laboral hacia la libre movilidad de personal laboral cualificado (ejecutivos, cuadros directivos, agentes comerciales, etc...). En este sentido, los excelentes tiempos de viaje que proporciona el TAV permiten más que nunca la descentralización espacial y la difusión de las actividades empresariales, pues la alta velocidad, complementada con el auge de las nuevas tecnologías, permiten una difusión de la información y de las innovaciones hasta el momento desconocida.

Sin embargo, la generalización de la movilidad al conjunto de la sociedad debe ser un objetivo irrenunciable. Es aquí donde entra en juego el concepto de rentabilidad social. Para que los efectos del TAV sean rentables tanto económica como socialmente se debe apostar por una política tarifaria que permita el acceso a este medio de transporte de amplios sectores de la sociedad. Se trata de hacer extensible las mejoras en la movilidad que supone el TAV al conjunto de la sociedad, y no sólo a los segmentos superiores de la demanda. De este modo, los efectos de la alta velocidad serán multiplicadores y redundarán en beneficio del conjunto de la sociedad. La aparente falta de rentabilidad de una política tarifaria blanda se ve pronto compensada con una utilización masiva del TAV por parte de la población, una vez que ésta comprueba las grandes ventajas del ahorro de tiempo en los desplazamientos que comporta.

En este sentido, la política seguida en Francia por la $\mathrm{SNCF}^{3}$ tiende a conseguir una utilización generalizada del TGV (Train à grande vitesse), por el conjunto de la sociedad francesa, en base a una política tarifaria caracterizada por unos precios asequibles y a la existencia de numerosos descuentos que se aplican en función de determinados criterios (menores de 26 años, jubilados, viajes en grupo, adquisición del billete con una antelación determinada, etc...). De este modo, los grupos caracterizados por una menor movilidad (jóvenes, jubilados, etc...) tienen mayores facilidades para desplazarse y se cumple la función social que debe tener el transporte público.

Esta utilización del TGV por el conjunto de la sociedad tiene, además, un efecto acumulador y permite a medio y largo plazo la rentabilidad en el establecimiento de relaciones ferroviarias con una gran frecuencia entre las ciudades, lo que implica una cobertura territorial y espacial del transporte ferroviario óptima. Ésta no se limita exclusivamente a las vías recorridas por el TGV, sino que extiende sus efectos a extensas áreas vertebradas por un tronco común (la línea en sí de alta velocidad), y numerosas ramificaciones que parten de él, representadas por las vías convencionales. Se consigue, de este modo, beneficiar de la alta velocidad ferroviaria a extensas áreas espaciales y no sólo a unos pocos focos demográficos y económicos importantes (ARDUIN, 1991).

Las autopistas. En el caso de las autopistas estamos ante un tipo de infraes-

\footnotetext{
${ }^{3}$ Societé National des Chemins de Fer, Compañía Pública de los Ferrocarriles Franceses.

${ }^{4}$ Tren de alta velocidad francés
} 
tructura caracterizada por una mayor capacidad de flujos de tráfico, y apta igualmente tanto para el transporte de personas como de mercancías. La mayor discrecionalidad del transporte viario, en relación con el carácter puerta a puerta de la movilidad y la libertad en la configuración de un itinerario determinado, así como el carácter abierto y flexible del transporte por carretera, han hecho del transporte por carretera-autopista el medio de transporte mayoritario no sólo en el transporte de mercancías, sino también en el de personas.

En el caso de la movilidad de personas, la generalización del automóvil privado, la construcción de densas redes de autopistas que permiten unas velocidades de $120-130 \mathrm{~km} / \mathrm{h}$ y, sobre todo, la libertad en cuanto a horarios y elección del desplazamiento que proporciona el automóvil privado han hecho del vehículo privado el medio más utilizado en los países desarrollados.

En cuanto al transporte de mercancías, a pesar de los mayores costes sociales que implica la movilización de mercancías por carretera (un solo tren puede transportar la misma cantidad de mercancías que varias decenas de camiones, con un solo conductor), éste se ha impuesto claramente al transporte ferroviario por las mismas razones que en el caso anterior: flexibilidad y discrecionalidad a la hora de la elección del intinerario; al mismo tiempo hay que tener en cuenta la mayor extensión de la red de carreteras y autopistas que la red ferroviaria, que se limita a los grandes corredores de circulación entre ciudades y otros núcleos de población de importancia.

Hoy en día los gobiernos de los países desarrollados apuestan por la construcción de tupidas redes de autopistas para poner en relación los principales núcleos de su sistema urbano (RoJO, 1995), conscientes de la importancia de las infraestructuras para el desarrollo del territorio; aunque la modernización de las redes ferroviarias es considerada prioritaria y se le empieza también a conceder especial importancia ${ }^{5}$, son las autopistas las que concentran la mayor parte de los esfuerzos inversores, como ha sucedido en primer lugar en USA y posteriormente en Europa, en donde España no es una excepción y la construcción de autopistas y autovías es considerada prioritaria, desde el Plan General de Carreteras 1984-1991, tanto por los poderes públicos como por la opinión pública antes que las infraestructuras ferroviarias. Una última cuestión hace referencia a la extensión y a las características de las áreas espaciales delimitadas por las autopistas y por el TAV. Se perciben también aquí las diferencias entre ambos medios de transporte, ya que si bien en ambos casos el área de influencia directa adopta una forma longitudinal, alargada y paralela en torno a la traza de la vía de comunicación, ambos casos presentan diferencias: más concentrada y discontinua en torno a los polos en el caso del TAV y más difusa y desconcentrada en el caso de las autopistas. Desde luego, esto implica unos efectos espaciales concretos, con más tendencia a polarizar el territorio y a acentuar la dualidad rural-urbano (favoreciendo sobre todo las ciudades en las cuales tiene parada el tren) en el caso del TAV, y con una tendencia más reequilibradora desde el punto de vista territorial en el caso de las autopis-

\footnotetext{
${ }^{5}$ El transporte ferroviario es, sin duda alguna, el menos contaminante y el más rentable desde el punto de vista del ahorro energético, por su no dependencia del petróleo.
} 
tas.

Ambos medios de transporte deben ser, por lo tanto, complementarios, y no cabe realizar interpretaciones excluyentes de la necesidad de invertir en uno u otro; la mayor diversidad modal contribuye a un aumento general de la movilidad y permite cubrir mayores huecos de la demanda de transporte, lo cual tiene indudablemente efectos beneficiosos para no sólo para el desarrollo económico del territorio, sino para lograr la cohesión social que los poderes públicos deben tratar de promover.

\section{GALICIA ANTE LAS NUEVAS INFRAESTRUCTURAS DE TRANSPORTE.}

Galicia, como región físicamente periférica en Europa y también dentro de España, iniciará el siglo XXI con graves carencias en cuanto a la dotación de infraestructuras de transporte, si bien es justo reconocer que en las últimas décadas se han producido grandes avances en lo que a infraestructuras de transportes se refiere.

Dejando al margen el ferrocarril, del cual hemos dicho ya que presenta una situación muy desfavorable, que podemos calificar como de enclave con respecto al resto de España, Galicia ha sido la última de las comunidades autónomas españolas en dotarse de las autovías diseñadas en el Plan General de Carreteras 19841991. Todavía en la actualidad, la autovía que une A Coruña con Madrid no está íntegramente finalizada. Por ello, está plenamente justificada la inversión, por parte de las distintas administraciones públicas (Xunta, Estado, UE), en infraestructuras de transporte modernas que sitúen a la región en pie de igualdad con otros territorios peninsulares, impulsando la llegada de la alta velocidad (o, en su defecto, la velocidad alta ${ }^{6}$ a Galicia y la finalización de las autovías de unión con la Meseta, así como la construcción de una serie de autopistas interiores estratégicas que aseguren una buena accesibilidad a todo el territorio gallego.

Ferrocarril $^{7}$. La llegada de la alta velocidad a Galicia hoy por hoy se asemeja más a una utopía que a una realidad. En el período de tiempo correspondiente al

\footnotetext{
${ }^{6}$ Se entiende por alta velocidad ferroviaria aquella que supera los $250 \mathrm{~km} / \mathrm{h}$ de media en líneas especialmente acondicionadas, mientras que la velocidad alta sitúa el intervalo entre los $150 \mathrm{y}$ los $250 \mathrm{~km} / \mathrm{h}$, pudiendo ser utilizadas en parte las vías convencionales con modificaciones en su trazado, tanto en geometría de planta como en cuanto a pendientes.

${ }^{7}$ NOTA DEL AUTOR: Cuando ya habíamos entregado el texto del presente artículo a la redacción de Polígonos, el día 21 de junio de 2000 el Ministerio de Fomento se comprometió con la Xunta de Galicia a la ejecución del ferrocarril de alta velocidad que unirá Madrid con Galicia en un plazo de siete años, con un montante global de inversión de 561.636 millones de pesetas.

En dicho acuerdo se contempla la solución sur (por Zamora) para la entrada de la alta velocidad en Galicia. El papel nodal de Ourense queda puesto de manifiesto, ya que este ciudad partirán los dos ramales que llegarán hasta A Coruña y Vigo.

Como se destaca en el artículo, la adopción de este trazado supone la marginación de buena parte de Galicia de la moderna red ferroviaria. Especialmente claro es en el caso de la provincia de Lugo, donde políticos y agentes sociales manifiestan abiertamente su oposición a una solución que, a la postre, agudizará los desequilibrios territoriales de la región, a no ser que se acompañe de actuaciones paralelas de mejora en la red ferroviaria convencional gallega, especialmente en la línea Monforte-Lugo-A Coruña.
} 
PDI, Plan Director de Infraestructuras (1993-2007), y en virtud de sendos acuerdos suscritos por la Xunta de Galicia con el Ministerio de Fomento y con RENFE, por un volumen de inversión de 30.000 y 63.597 millones de pesetas, respectivamente, tan sólo se contemplan pequeñas reducciones de los tiempos de viaje.

Se trata sobre todo de corrección de trazados en planta mediante la construcción de variantes, con especial atención al eje ferroviario A Coruña-Vigo, al cual se destinarán en total 42.247 millones de pesetas. Los accesos a Galicia desde la Meseta y la conexión con Portugal también recibirán inversiones, menos cuantiosas en este caso, destinadas a la mejora y el acondicionamiento de las infraestructuras ferroviarias.

En todos los casos, estamos ante actuaciones consistentes en parches en la red ferroviaria actual. Aunque después de realizadas las inversiones (año 2007) se conseguirán velocidades competitivas, en torno a los $160 \mathrm{~km} / \mathrm{h}$ en tramos concretos, el conjunto de la red seguirá siendo de vía única y sin electrificar (a excepción del tramo Monforte-Ourense-Vigo ya electrificado). Asimismo, nada se dice acerca de la llegada de la alta velocidad o velocidad alta a Galicia. Tan solo se sabe que la AV llegará a Valladolid, tal y como se recoge en el PDI en un futuro más o menos próximo, tramo que incluye la ejecución de la controvertida Variante del Guadarrama. A partir de Valladolid, responsables políticos de la Xunta han hecho declaraciones alusivas a la llegada de la alta velocidad a Monforte de Lemos (que se recoge, asimismo, en los primeros documentos de redes transeuropeas de alta velocidad), pero lo cierto es que no hay nada en firme acerca de la llegada al menos de la velocidad alta a Galicia.

Sin embargo, la llegada "sine die" de la AV ha provocado las primeras discusiones sobre cuál sería el trazado más idóneo de acceso a Galicia. De nuevo se han reavivado viejas polémicas entre el norte y el sur de la comunidad, que en su día retrasaran la llegada del primer ferrocarril a Galicia.

Las dos opciones que se barajan son la entrada de la AV por Monforte-Ourense o a la entrada por las Zamora-Verín-Ourense. En ambos casos, la conexión con el Eje Urbano Atlántico y con los principales centros urbanos, demográficos y económicos de la región está asegurada, en virtud del elevado volumen de inversión que privilegiará las obras en el Eje Atlántico y conseguirá unos tiempos de viajes competitivos.

Sin embargo, con la ejecución de cualquiera de los dos trazados resulta enormemente marginado un espacio que se corresponde con la Mariña y, en general, el Noreste de Lugo (ver FIGURA № 2). Esta área quedaría muy mal servida por ferrocarril, no sólo por no transcurrir por él el trazado de la alta velocidad, sino por las deficientes condiciones que presenta la infraestructura férrea en la actualidad entre Monforte-Lugo-Betanzos y entre Betanzos-Ferrol.

La llegada de la alta velocidad a Galicia deberá tener en cuenta, por lo tanto, la existencia de esta extensa área, y venir acompañada de una modernización de la infraestructura ferroviaria y del material rodante existente que permita al norte de Galicia comunicarse eficazmente con Monforte u Ourense, futuros nodos de enlace con la línea de alta velocidad que unirá Galicia con la Meseta.

Autopistas. La situación por lo que se refiere a la dotación de autopistas es sensiblemente mejor, ya que ha sido la carretera (por otra parte, el medio de trans 
Fig. $\mathrm{n}^{\circ} 2$ : Principal área con déficit de accesibilidad a la futura red de alta velocidad gallega

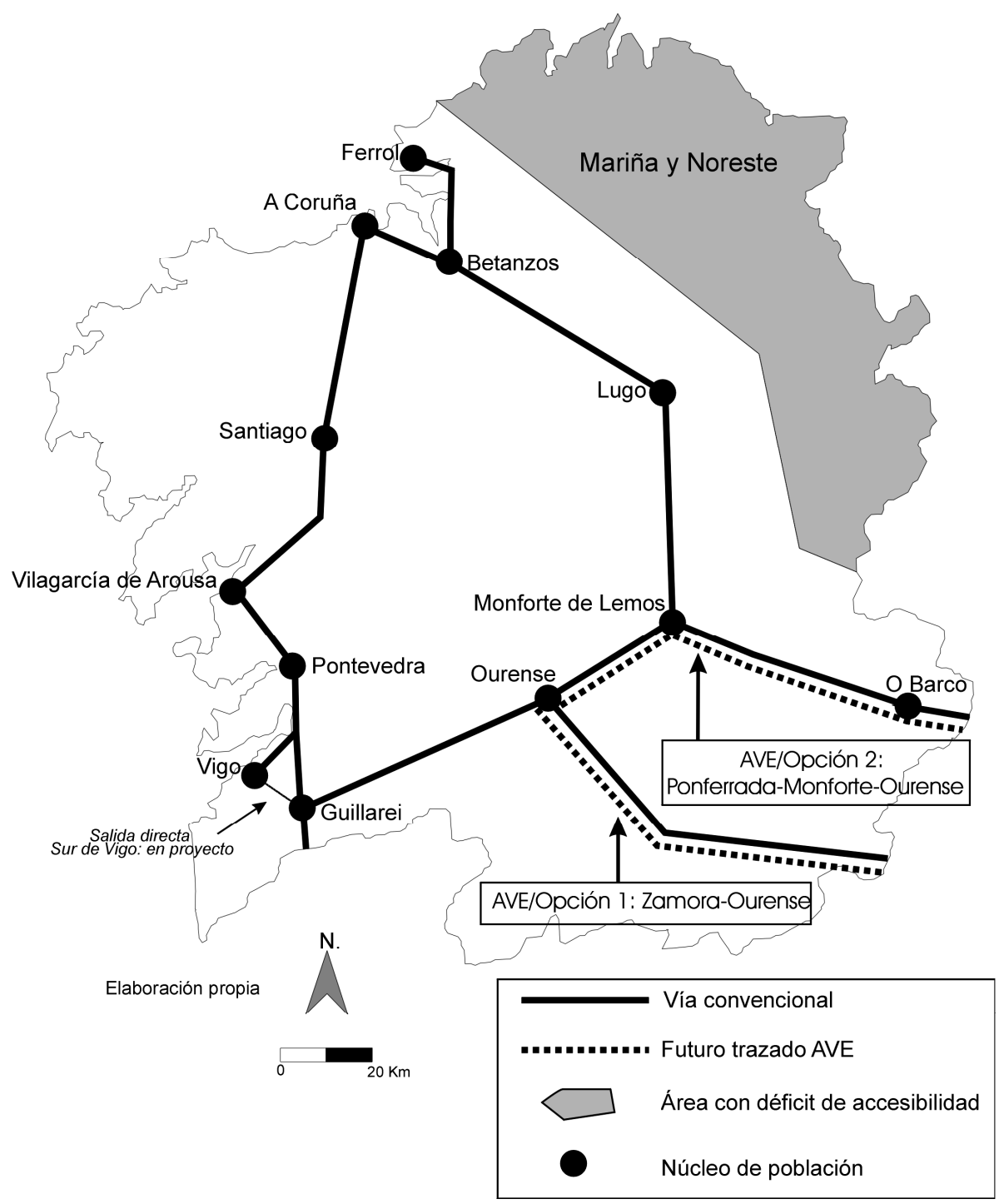


porte con mayor importancia en la actualidad, con mucha diferencia), la elegida por los poderes públicos, tanto regionales como estatales, para acometer la urgente y necesaria modernización de las infraestructuras de transporte terrestre que se pone en marcha tras el Plan General de Carreteras (1984-1991), y que ya se había comenzado en la década de los 70 con el inicio de la construcción de la Autopista del Atlántico. Sin embargo, como ya ha sido puesto de manifiesto (PAZOS OTÓN, 1999), la red de autopistas y autovías gallega responde a una concepción estática del planeamiento territorial que puede tener como efecto más grave la agudización y la potenciación de los desequilibrios territoriales ya existentes.

En efecto, dejando a parte el Eje Urbano Atlántico, vertebrado por la Autopista del Atlántico (la primera vía de alta capacidad comenzada a ejecutar en Galicia), y complementado por las autopistas A Coruña-Carballo y Vigo-Baiona, de titularidad autonómica, el resto del territorio gallego está únicamente servido por dos vías de alta capacidad, las Autovías del Noroeste y las Rías Baixas. Éstas comunican Madrid con las dos cabeceras del sistema urbano gallego, Vigo y A Coruña, y reproducen de nuevo los esquemas radiales y centralistas de planeamiento territorial español en materia de infraestructuras, hecho común a toda la historia de las redes modernas de infraestructuras modernas.

No se trata de unas autovías pensadas y planificadas para la vertebración interna de Galicia, sino que éstas tratan de dar respuesta únicamente al gran déficit de accesibilidad de Galicia con el resto de España, puesto de manifiesto y objetivo declarado del PDI. A pesar de que se integran en la red de alta capacidad las capitales del interior (Lugo y Ourense), las autovías por sí solas no cumplen un papel estructurante del interior de Galicia, ya que quedan amplias áreas vacías sin cobertura y mal comunicadas a través de una vía de alta capacidad (ver FIGURA N ${ }^{\circ}$ 3). Éste es el caso, entre otros, de las áreas siguientes:

- Centro e interior de Galicia. Las comunicaciones de Santiago con Ourense y de Ourense con Lugo por autopista aún tendrán que esperar. En el primer caso, se ha adjudicado sólo la primera mitad de la obra, entre Santiago y el Alto de Santo Domingo, en un corredor que registra una alta movilidad y donde es necesaria la autopista debido a la saturación que presenta la carretera N-525. En el caso de la autopista Lugo-Ourense, de la cual se habló mucho durante el período de constitución de la Autonomía, ha quedado en el olvido, a pesar de ser una infraestructura estratégica, enmarcada dentro del Eje Interior gallego, que vertebraría el interior de Galicia y actuaría de contrapeso al Eje Urbano Atlántico.

- Noreste de Galicia y, en especial, la Mariña Lucense. El aislamiento del N. de Galicia y de la Mariña Lucense en particular es sensible y se percibe en la actualidad en el hecho de que Ferrol todavía no esté conectado a la red de alta capacidad gallega. La distancia y la mala accesibilidad con respecto a la Autovía del Noroeste y la incertidumbre con respecto al denominado Corredor Transcantábrico, del que no se conoce ni su trazado definitivo ni su fecha de ejecución ni finalización, son la tónica en un área con grandes potencialidades si mejora su accesibilidad con las comunidades cantábricas (lo que supone la salida directa hacia Europa) y con el Corredor Atlántico. 
Fig. $\mathrm{n}^{\circ}$ 3: Principales áreas con déficit de accesibilidad a la red viaria de alta capacidad gallega

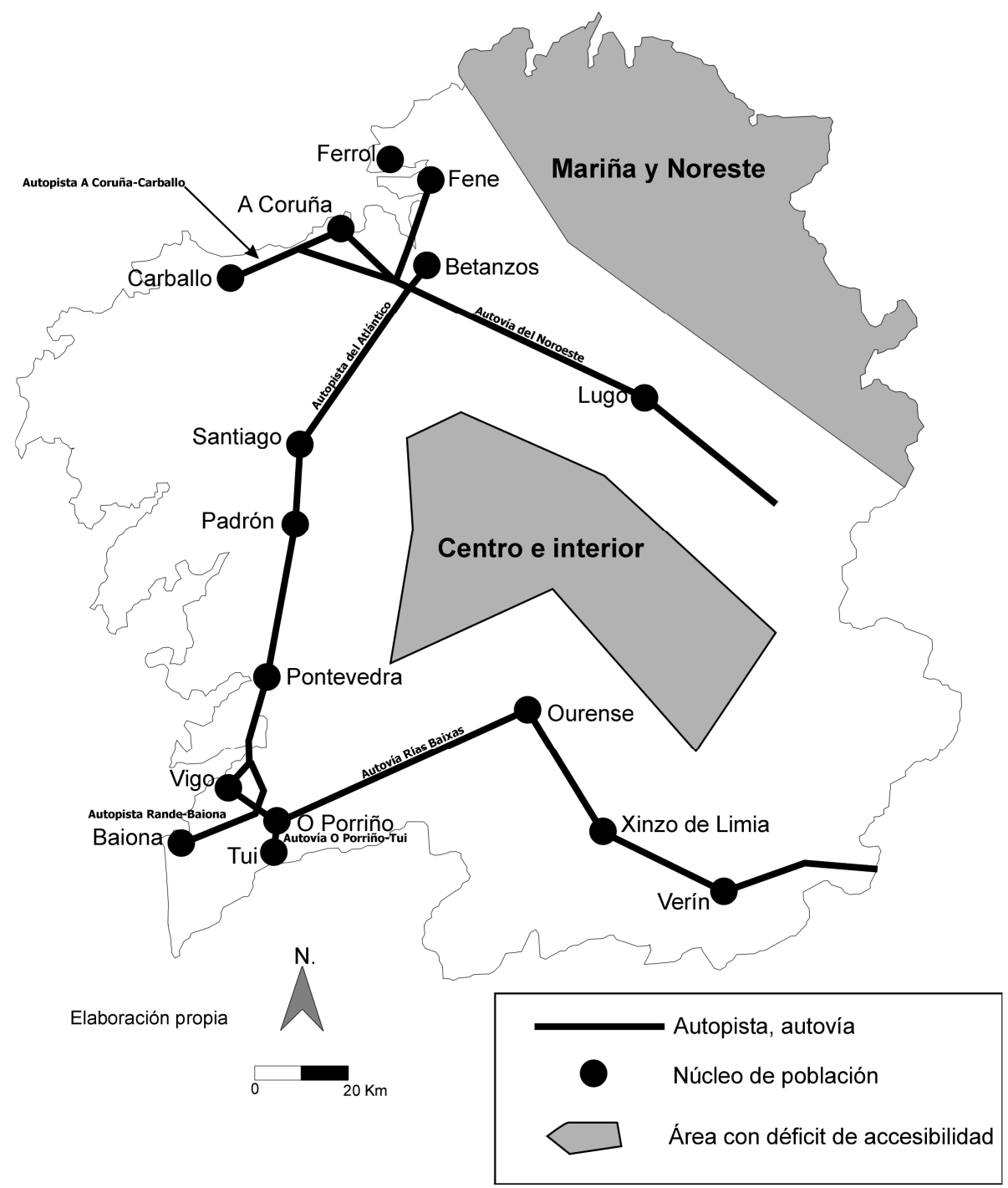




\section{CONCLUSIONES.}

En el sistema económico de mercado en el que nos hallamos inmersos, es cada vez más importante la movilidad de personas, mercancías e información, como un elemento clave del proceso productivo. En este sentido, los grandes cambios espaciales producidos a raíz de la crisis del modelo de acumulación industrial y la descentralización del proceso productivo conceden a los transportes un papel clave, reforzado aún más si cabe por el auge de métodos de producción como el justin-time y la eliminación de stocks, lo que convierte al transporte en un factor estratégico para el sistema económico.

En este contexto, los estados y las regiones deben fomentar la articulación territorial gracias a nuevas infraestructuras de transporte terrestres. Por las características de la movilidad actual, estas nuevas infraestructuras vienen representadas por las redes de autopistas y el TAV, dos elementos estratégicos que presentan características y repercusiones territoriales similares y a la vez diferentes, y que políticos y planificadores territoriales deben manejar con sumo cuidado para conseguir un desarrollo armónico del territorio y evitar la aparición y el agravamiento de los temibles desequilibrios territoriales.

El caso gallego, por su perificidad, su situación excéntrica en España y en Europa y por la deficiente base de infraestructuras existentes heredada del pasado, es especialmente representativo de un territorio periférico de la UE que precisa conectarse con las redes europeas de autopistas y alta velocidad. Pero este desarrollo, necesario para que Galicia no pierda el tren del progreso y pueda competir en pie de igualdad con otros territorios, no debe ser alcanzado a toda costa.

Antes bien, la llegada de las nuevas infraestructuras debe ser aprovechada asimismo para lograr la vertebración y cohesión territorial interna del conjunto de Galicia, aspecto que, lejos de conseguirse, está agudizándose hoy en día, con la potenciación de los desequilibrios entre el litoral y el interior, siendo éste el gran olvidado en las políticas de infraestructuras.

Así, las actuaciones futuras de autopistas y TAV en Galicia deberán tener en cuenta el riesgo de marginalización y enclavamiento por lo que se refiere a la accesibilidad de extensos territorios. Una distribución más equitativa de las inversiones, en el caso de las autopistas, y la modernización de la red convencional para su conexión con la red de alta velocidad ferroviaria son aspectos que, a nuestro juicio, deberán tenerse en cuenta en el futuro para no repetir errores cometidos en el pasado.

\section{BIBLIOGRAFÍA.}

- ARDUIN, Jean-Pierre (1991): «Las líneas de alta velocidad y el acondicionamiento del territorio». En: Revista Obras Públicas, n 22, pp. 22-33. Madrid.

- BeIRAS Torrado, X.M. (1972): O atraso económico de Galicia. Ed. Galaxia. Vigo. 209 pp.

- BIEHL, D. (1989): «El papel de la Infraestructura en el desarrollo regional». En: Política Regional en la Europa de los años 90. Ministerio de Economía y Hacienda. Madrid. 
- LOBO GUTIÉRREZ, Ignacio (1993): «Carreteras y desarrollo económico: España, diciembre de 1993». En: Razón y Fe, no 145, pp. 263-285. Ed. Herder. Barcelona.

- MerLin, P. (1991): Géographie, Économie et Planification des Transports. Presses Universitaires de France. 473 pp.

- MERLIN, Pierre (1992): «Géographie, économie et planification des transports». En: Recherche Transports Sécurité, $\mathrm{n}^{\circ} 33$, pp. 70-72. Edita: INRET. Paris.

- MASSEY, D. (1994): Space, Place and Gender. Polity Press. Cambridge. 208 pp.

- NóVOA RodRíGUEZ, Xoán (1991): «El Noroeste: Estación Término». En: Revista Obras Públicas, $\mathrm{n}^{\mathrm{o}}$ 22, pp. 78-97. Madrid.

- PAZOS OTÓN, M. (1999): «Las infraestructuras internas de transporte en Galicia y su influencia en los desequilibrios territoriales». Comunicación al I Seminario sobre perspectivas de desarrollo en Iberoamérica, pp. 229-241. Libro de Actas. Universidade de Santiago.

- PÉREZ TOURIÑO, E. (1992): «Las infraestructuras como factor de despegue del desarrollo gallego». En: GONZÁLEZ LAXE, F (dir.): Estructura Económica de Galicia, pp.429-488. Espasa Calpe. Madrid.

- QUINET, E. (1992): Infrastructures de Transport et Croissance. Ed. Económica. París. 123 pp.

- RoJo, Antonio (1995): «Las redes de autopistas transeuropeas». En: Política Exterior, ${ }^{\circ}$ 45, pp. 62-80. Madrid.

- VV.AA. (1994): Redes Transeuropeas. Edita: Comisión Europea. Bruselas.

- VV.AA. (1999): «Plan de inversiones en infraestructura para la red gallega». En: Líneas del tren, $\mathrm{n}^{\circ}$ 193, pp. 13-16. Edita: RENFE. Dirección de Comunicación Interna. Madrid. 